\title{
On the complexity of sequentially lifting cover inequalities for the knapsack polytope
}

\author{
Wei-Kun Chen · Yu-Hong Dai*
}

March 12, 2019

\begin{abstract}
The well-known sequentially lifted cover inequality is widely employed in solving mixed integer programs. However, it is still an open question whether a sequentially lifted cover inequality can be computed in polynomial time for a given minimal cover (Gu, Nemhauser, and Savelsbergh, INFORMS J. Comput., 26: 117-123, 1999). We show that this problem is $\mathcal{N} \mathcal{P}$-hard, thus giving a negative answer to the question.
\end{abstract}

Keywords Integer programming · Sequentially lifted cover inequality · Complexity $\cdot$ Lifting problem

Mathematics Subject Classification (2000) 90C11 - 90C27

\section{Introduction}

The lifted cover inequality (LCI) is a well-known cutting plane for mixed integer programs. Given the so-called cover inequality, in order to obtain an LCI, we may use the lifting technique. Using different lifting procedures, several types of LCIs have been studied in the literatures, see 1, 3, 4, 11, 13, 14, 19, 24. In this paper, we are concentrated on the sequential LCI, that is, the variables are sequentially lifted one at a time. The sequential LCI was first studied in [21, 24]. Its effectiveness on using as a cutting plane was demonstrated in [6], see also 10, 15, 16, 23, 25]. To lift each variable, a knapsack problem is required to be solved to compute the lifting coefficient. Under certain conditions, the sequential LCI can be computed in polynomial time, see [8, 20, 26]. In general,

This work was supported by the Chinese Natural Science Foundation (Nos. 11631013, 11331012) and the National 973 Program of China (No. 2015CB856002).

* Corresponding author

LSEC, ICMSEC, Academy of Mathematics and Systems Science, Chinese Academy of Sciences, Beijing, China - School of Mathematical Sciences, University of Chinese Academy of Sciences, Beijing, China. E-mail: cwk@lsec.cc.ac.cn · dyh@lsec.cc.ac.cn 
however, the complexity of computing a sequential LCI is still unknown. This was explicitly mentioned in [9] as an open question.

"We show that this dynamic programming algorithm may take exponential time to compute a sequential LCI that is not simple. It is still an open question whether an arbitrary LCI can be computed in polynomial time for a given minimal cover $C$."

The above question was also cited as an open question in [2, 8, 12, 22]. We will give a negative answer to the question by showing that the problem of computing a sequential LCI is $\mathcal{N} \mathcal{P}$-hard. Thus, unless $\mathcal{P}=\mathcal{N} \mathcal{P}$, there exists no polynomial time algorithm to compute a sequential LCI.

This paper is organized as follows. In Section 2 we review how to compute a sequential LCI. In Section [3, we describe the elegant example by Gu 8], which provides exponential lifting coefficients. The main result is given in the last section, which shows the $\mathcal{N} \mathcal{P}$-hardness of the problem of computings a sequential LCI.

\section{Computing a sequentially lifted cover inequality}

Consider the knapsack set $X:=\left\{x \in \mathbb{B}^{n}: a^{T} x \leq b\right\}$, where $b \in \mathbb{Z}_{+}$and $a=\left(a_{1}, \cdots, a_{n}\right)^{T} \in \mathbb{Z}_{+}^{n}$ are given. A subset $C \subseteq N:=\{1, \ldots, n\}$ is called a cover of $X$ if the sum of the items $a_{i}$ 's over $C$ exceeds the knapsack capacity $b$; i.e., $\sum_{i \in C} a_{i}>b$. A cover $C$ is a minimal cover if and only if

$$
\sum_{i \in C \backslash\{j\}} a_{i} \leq b \quad \text { for all } j \in C .
$$

For any subsets $N_{0}$ and $N_{1}$ of $N$ with $N_{0} \cap N_{1}=\varnothing$, denote $X\left(N_{0}, N_{1}\right)$ be the following restriction set of $X$,

$$
X\left(N_{0}, N_{1}\right)=X \cap\left\{x \in \mathbb{B}^{n}: x_{i}=0 \text { for } i \in N_{0} ; x_{i}=1 \text { for } i \in N_{1}\right\} .
$$

It is easy to see that, for each cover $C$, the cover inequality

$$
\sum_{i \in C} x_{i} \leq c-1
$$

is valid for $X(N \backslash C, \varnothing)$, where $c:=|C|$ is the cardinality of $C$. The cover inequality (1) is facet defining for $\operatorname{conv}(X(N \backslash C, \varnothing))$, which is the convex hull of $X(N \backslash C, \varnothing)$, if and only if $C$ is a minimal cover (see for example [5]).

We can consider to fix some variables to be ones as well. Assume that $\left(C, N_{0}, N_{1}\right)$ is a partition of $N$ and denote $\bar{b}=b-\sum_{i \in N_{1}} a_{i}$. In this case, the inequality (11) is facet defining for $\operatorname{conv}\left(X\left(N_{0}, N_{1}\right)\right)$ if and only if $C$ is a minimal cover of $X\left(N_{0}, N_{1}\right)$; i.e.,

$$
\sum_{i \in C} a_{i}>\bar{b} ; \quad \sum_{i \in C \backslash\{j\}} a_{i} \leq \bar{b} \quad \text { for all } j \in C .
$$


Throughout this paper, we shall assume that $\left(C, N_{0}, N_{1}\right)$ be a partition of $N$ and $C$ is a minimal cover of $X\left(N_{0}, N_{1}\right)$.

In general, however, the inequality (10) may not be valid for $X$ if $N_{1} \neq \varnothing$. Furthermore, even if $N_{1}=\varnothing$, such an inequality may not represent a facet of $\operatorname{conv}(X)$. To obtain a strong valid inequality, we can lift the variables in $N_{0} \cup N_{1}$ one at a time by solving an optimization problem sequentially. More precisely, let $l_{1}, \ldots, l_{n-c}$ be a lifting sequence such that $N_{0} \cup N_{1}=\left\{l_{1}, \ldots, l_{n-c}\right\}$ and

$$
\sum_{i \in C} x_{i}+\sum_{i \in N_{0}^{\prime}} \alpha_{i} x_{i}+\sum_{i \in N_{1}^{\prime}} \beta_{i} x_{i} \leq c-1+\sum_{i \in N_{1}^{\prime}} \beta_{i}
$$

be the inequality obtained so far, where $N_{0}^{\prime} \subseteq N_{0}, N_{1}^{\prime} \subseteq N_{1}$ and $N_{0}^{\prime} \cup N_{1}^{\prime}=$ $\left\{l_{1}, \ldots, l_{j}\right\}$. To lift the variable $x_{l_{j+1}}$, we need to solve a knapsack subproblem depending on whether $l_{j+1}$ belongs to $N_{0}$ or $N_{1}$. We follow [10] in referring to lifting the variable $x_{l_{j+1}}$ as up-lifting if $l_{j+1} \in N_{0}$ and down-lifting if $l_{j+1} \in N_{1}$.

(i) Up-lifting. If $l_{j+1} \in N_{0}$, compute the lifting coefficient $\alpha_{l_{j+1}}$ by solving the lifting problem

$$
\begin{aligned}
& \alpha_{l_{j+1}}=\min \quad c-1+\sum_{i \in N_{1}^{\prime}} \beta_{i}-\sum_{i \in C} x_{i}-\sum_{i \in N_{0}^{\prime}} \alpha_{i} x_{i}-\sum_{i \in N_{1}^{\prime}} \beta_{i} x_{i} \\
& \text { s.t. } \quad \sum_{i \in C \cup N_{0}^{\prime} \cup N_{1}^{\prime}} a_{i} x_{i} \leq \bar{b}+\sum_{i \in N_{1}^{\prime}} a_{i}-a_{l_{j+1}}, x \in \mathbb{B}^{c+j} \text {. }
\end{aligned}
$$

(ii) Down-lifting. If $l_{j+1} \in N_{1}$, compute the lifting coefficient $\beta_{l_{j+1}}$ by solving the lifting problem

$$
\begin{aligned}
\beta_{l_{j+1}}=\max & \sum_{i \in C} x_{i}+\sum_{i \in N_{0}^{\prime}} \alpha_{i} x_{i}+\sum_{i \in N_{1}^{\prime}} \beta_{i} x_{i}-c+1-\sum_{i \in N_{1}^{\prime}} \beta_{i} \\
\text { s.t. } & \sum_{i \in C \cup N_{0}^{\prime} \cup N_{1}^{\prime}} a_{i} x_{i} \leq \bar{b}+\sum_{i \in N_{1}^{\prime}} a_{i}+a_{l_{j+1}}, x \in \mathbb{B}^{c+j} .
\end{aligned}
$$

After having lifted all the variables, we obtain the sequential LCI:

$$
\sum_{i \in C} x_{i}+\sum_{i \in N_{0}} \alpha_{i} x_{i}+\sum_{i \in N_{1}} \beta_{i} x_{i} \leq c-1+\sum_{i \in N_{1}} \beta_{i} .
$$

The procedure to obtain inequality of (4) is first described implicitly in 24]. See for example [8, 17, 20] for more details about sequential LCI. Here we just notice that different lifting sequences may lead to different sequential LCIs.

The inequality (4) is called a non-project LCI if $N_{1}=\varnothing$ and a project LCI if $N_{1} \neq \varnothing$ (see [8]). Given a lifting sequence, the non-project LCI can be computed $([26])$ in the complexity of $\mathcal{O}(c n)$, where $c=|C|$ again. For the project LCI, if $C \cup N_{1}$ is a minimal cover of $X$ and the lifting sequence is enforced to $\left\{l_{1}^{0}, \ldots, l_{r}^{0}, l_{1}^{1}, \ldots, l_{\left|N_{1}\right|}^{1}, l_{r+1}^{0}, \ldots, l_{\left|N_{0}\right|}^{0}\right\}$, where $l_{i}^{j} \in N_{j}$ for $i=1, \ldots,\left|N_{j}\right|$ and $j=0,1$ and $r$ is a given integer between 1 and $\left|N_{0}\right|, \mathrm{Gu}$ [8] proved that (4) can be obtained in the complexity of $\mathcal{O}\left(\mathrm{cn}^{3}\right)$. As mentioned before, however, the 
complexity of computing a sequential LCI with an arbitrary lifting sequence is still unknown.

We close this section by noting that the sequential LCI is invariant under scaling.

Observation 1 Given the same partition and the lifting sequence, multiplying a positive integer to the knapsack constraint gives the same sequential LCI.

\section{The example by Gu (1995)}

In this section, we describe the elegant example constructed by $\mathrm{Gu}$ [8], which leads the lifting coefficients to be exponential. It is related to the following vector $f \in \mathbb{Z}^{2 r+1}$, where $r$ is a given positive integer.

$$
f_{1}=1, f_{2}=1, f_{3}=1 \text {, and } f_{i}=f_{i-2}+f_{i-1}, \text { for } i=4, \ldots, 2 r+1 \text {. }
$$

Notice that $f$ is analogous to the Fibonacci sequence where the only difference is that the first element in the Fibonacci sequence is 0 . We give two facts on the vector $f$, which can easily be verified by induction.

Observation 2 For $j=3, \ldots, 2 r+1$, it holds that $f_{j}=\sum_{i=1}^{j-2} f_{i}$.

Observation 3 For $j=3, \ldots, 2 r+1$, it holds that $\frac{1}{4}(\sqrt{2}-1)(\sqrt{2})^{j} \leq f_{j} \leq 2^{j}$.

Consider the knapsack set $X$ with $2 r+1$ variables, where the coefficients of the knapsack constraint are $f_{1}, \ldots, f_{2 r+1}$ in (5) and the associated knapsack capacity is $b=\sum_{i=1}^{2 r} f_{i}$. Consider the partition $\left(C, N_{0}, N_{1}\right)$ of $\{1, \ldots, 2 r+1\}$ with $C=\{1,2\}, N_{0}=\{4,6,8, \ldots 2 r\}$, and $N_{1}=\{3,5,7, \ldots, 2 r+1\}$. Since $f_{1}=1, f_{2}=1$, and

$$
\begin{aligned}
\bar{b} & =b-\sum_{i \in N_{1}} f_{i}=b-\sum_{i=1}^{r} f_{2 i+1}=b-f_{3}-\sum_{i=2}^{r}\left(f_{2 i-1}+f_{2 i}\right) \\
& =b-f_{3}-\sum_{i=3}^{2 r} f_{i}=1
\end{aligned}
$$

we know that $C$ is a minimal cover of $X\left(N_{0}, N_{1}\right)=\left\{x \in \mathbb{B}^{2}: x_{1}+x_{2} \leq 1\right\}$. Now consider the lifting sequence $\{3,4, \ldots, 2 r+1\}$; i.e., $l_{j}=j+2$ for $1 \leq$ $j \leq 2 r-1$. The following lemma is due to [8]. For completeness, we provide a proof.

Lemma 1 (Gu 1995) (i) $\alpha_{i}=f_{i}$ for $i \in N_{0}$; (ii) $\beta_{i}=f_{i}$ for $i \in N_{1}$.

Proof. We proceed by induction. At first, the lifting problem of the variable $x_{3}$ reads

$$
\begin{aligned}
\beta_{3}=\max & x_{1}+x_{2}-1 \\
\text { s.t. } & x_{1}+x_{2} \leq 2, x \in \mathbb{B}^{2} .
\end{aligned}
$$


Hence $\beta_{3}=1=f_{3}$. Assume that $\alpha_{i}=f_{i}$ for $i \leq j, i \in N_{0}$ and $\beta_{i}=f_{i}$ for $i \leq j, i \in N_{1}$, respectively. Now we consider lifting the variable $x_{j+1}$. If $j+1 \in N_{0}$, the problem (2) reduces to

$$
\begin{aligned}
\alpha_{j+1}= & \min \quad 1+\sum_{i=1}^{(j-1) / 2} f_{2 i+1}-\sum_{i=1}^{j} f_{i} x_{i} \\
\text { s.t. } & \sum_{i=1}^{j} f_{i} x_{i} \leq 1+\sum_{i=1}^{(j-1) / 2} f_{2 i+1}-f_{j+1}, x \in \mathbb{B}^{j} .
\end{aligned}
$$

Since

$$
\sum_{i=1}^{(j-1) / 2} f_{2 i+1}=f_{3}+\sum_{i=2}^{(j-1) / 2}\left(f_{2 i}+f_{2 i-1}\right)=f_{3}+\sum_{i=3}^{j-1} f_{i}=\sum_{i=1}^{j-1} f_{i}-1=f_{j+1}-1,
$$

where the last equality follows from Observation 2. the feasibility of the problem (6) requires $x_{i}=0,1 \leq i \leq j$ and hence $\alpha_{j+1}=f_{j+1}$. If $j+1 \in N_{1}$, the problem (3) reduces to

$$
\begin{aligned}
\beta_{j+1}= & \max \quad \sum_{i=1}^{j} f_{i} x_{i}-1-\sum_{i=1}^{j / 2-1} f_{2 i+1} \\
\text { s.t. } & \sum_{i=1}^{j} f_{i} x_{i} \leq 1+\sum_{i=1}^{j / 2-1} f_{2 i+1}+f_{j+1}, x \in \mathbb{B}^{j} .
\end{aligned}
$$

It is easy to verify that $\sum_{i=1}^{j} f_{i}=1+\sum_{i=1}^{j / 2-1} f_{2 i+1}+f_{j+1}$. Hence the all-one vector is feasible and solves the problem (7), yielding $\beta_{j+1}=f_{j+1}$. So the statement is true for $j+1$ as well. By induction, this lemma holds.

Lemma 1 indicates that the sequential LCI for this specific example is

$$
\sum_{i=1}^{2 r+1} f_{i} x_{i} \leq \sum_{i=1}^{2 r} f_{i}
$$

By Observation 3, the input size of this example is polynomial, but the lifting coefficients $\left\{f_{i}\right\}$ are exponential with $r$. This example by $\mathrm{Gu}$ will play an important role in the coming complexity analysis.

\section{$4 \mathcal{N} \mathcal{P}$-hardness of computing a sequentially lifted cover inequality}

In this section, we show the $\mathcal{N} \mathcal{P}$-hardness of the problem of computing a sequential LCI. To begin with, we give a basic property of the vector $f$ in (5) .

Lemma 2 Let $f$ be defined as in (5), where $r \geq 1$ is given. For any $\tau \in \mathbb{Z}_{+}$ satisfying $0 \leq \tau \leq \sum_{i=1}^{2 r+1} f_{i}$, there exists a subset $S \subseteq\{1, \ldots, 2 r+1\}$ such that $\tau=\sum_{i \in S} f_{i}$. 
Proof. We proceed by induction on $r$. The result apparently holds for $r=1$. Assume that the result is true for some $r \geq 1$. To verify the result for $r+1$, it suffices to consider the case that $\sum_{i=1}^{2 r+1} f_{i}<\tau \leq \sum_{i=1}^{2(r+1)+1} f_{i}$. In fact, from Observation 2, we have that

$$
\sum_{i=1}^{2 r+1} f_{i}=f_{2 r+3}, \quad \sum_{i=1}^{2 r+3} f_{i}=f_{2 r+3}+f_{2 r+2}+f_{2 r+3} .
$$

So $f_{2 r+3}<\tau \leq f_{2 r+3}+f_{2 r+2}+f_{2 r+3}$. Let us define $\tau_{1}$ as

$$
\tau_{1}= \begin{cases}\tau-f_{2 r+2}-f_{2 r+3}, & \text { if } \tau>f_{2 r+2}+f_{2 r+3} \\ \tau-f_{2 r+3}, & \text { if } \tau \leq f_{2 r+2}+f_{2 r+3} .\end{cases}
$$

Then it is easy to see that $\tau_{1} \leq f_{2 r+3}=\sum_{i=1}^{2 r+1} f_{i}$. By the induction assumption, there exists $S \subseteq\{1,2,3, \ldots, 2 r+1\}$ such that $\tau_{1}=\sum_{i \in S} f_{i}$. By picking one more element $f_{2 r+3}$ and the possible element $f_{2 r+2}$, we know that there exists some subset of $\{1,2,3, \ldots, 2 r+3\}$ such that the sum of $f_{i}$ 's over this subset is exactly $\tau$. Thus the result holds for $r+1$. By induction, this lemma is true.

Next, we introduce the restricted partition problem (RPP), which is a variant of the partition problem 7]. Comparing to the partition problem, the RPP problem restricts the total sum of all element to some specific values and allows the sum of the elements in the subset equals to one more value. The $\mathrm{RPP}$ problem is shown to be $\mathcal{N} \mathcal{P}$-hard in [18].

Problem RPP. Given a nonnegative integer $m$, a finite set $K$ of $k$ elements with value $\omega_{i} \in \mathbb{Z}_{+}$for the $i$-th element and $\sum_{i \in K} \omega_{i}=$ $2\left(2^{m+1}-1\right)$, does there exist a subset $T \subseteq K$ such that $\sum_{i \in T} \omega_{i}=$ $2^{m+1}-1$ or $\sum_{i \in T} \omega_{i}=2^{m+1}-2 ?$

For convenience, define $\lambda=2^{m+1}-1$ and then $\sum_{i \in K} \omega_{i}=2 \lambda$. The RPP problem is still $\mathcal{N} \mathcal{P}$-hard when $1 \leq \omega_{i} \leq \lambda-1$. To see this, suppose there exists some $j \in K$ such that $\omega_{j} \geq \lambda$. We have the following two cases.

(i) $\lambda \leq \omega_{j} \leq \lambda+1$. In this case, it follows that $\sum_{i \in K \backslash\{j\}} \omega_{i}=\lambda-1$ or $\sum_{i \in K \backslash\{j\}} \omega_{i}=\lambda$. Thus, $K \backslash\{j\}$ is the desired subset and the answer to the $\mathrm{RPP}$ problem is yes.

(ii) $\omega_{j} \geq \lambda+2$. In this case, it is easy to see that the answer to the RPP problem is no.

As we can see, both cases can be solved in polynomial time. Therefore, the statement follows and in the following, we assume that $1 \leq \omega_{i} \leq \lambda-1$. We are now ready to present the main result of this paper; i.e., provide an $\mathcal{N} \mathcal{P}$ hardness proof for computing a sequential LCI. The basic idea is as follows. Firstly, we adopt Gu's example (see Section 3) to make the lifting coefficients exponential. Secondly, some variables fixed at zero will be lifted, where the lifting coefficients can easily be obtained. Finally, we lift a variable fixed at one, where the objective value of corresponding lifting problem is equal to some specific value if and only if the answer to the RPP problem is yes and hence is $\mathcal{N} \mathcal{P}$-hard. 
Theorem 1 The problem of computing a sequential LCI is $\mathcal{N} \mathcal{P}$-hard.

Proof. For an RPP instance with $m \geq 0$ and $K=\{2 r+4, \ldots, n-1\}$, we shall construct a problem of computing a sequential LCI in polynomial time. We construct a problem of computing a sequential LCI as follows. Set $r=m+6$, $n=2 r+4+k$, and $b=\sum_{i=1}^{2 r+1} \lambda f_{i}+\lambda(3 \lambda+6)$, where $f$ is defined as in (5). Set the coefficients of the knapsack constraint as

$$
a_{i}= \begin{cases}\lambda f_{i}, & \text { for } i=1, \ldots, 2 r+1 \\ \lambda(\lambda+3)+1, & \text { for } i=2 r+2 \\ \lambda(\lambda+3)-1, & \text { for } i=2 r+3 \\ \omega_{i}(\lambda+1), & \text { for } i=2 r+4, \ldots, n-1 ; \\ \lambda\left(3 \lambda+6+f_{2 r+1}\right), & \text { for } i=n .\end{cases}
$$

Define the partition $\left(C, N_{0}, N_{1}\right)$ with $C=\{1,2\}, N_{0}=\{4,6,8, \ldots, 2 r, 2 r+$ $2,2 r+3, \ldots, n-1\}$, and $N_{1}=\{3,5,7, \ldots, 2 r+1, n\}$. Finally, let the lifting sequence be $\{3, \ldots, n\}$. We shall prove that the lifting coefficient $\beta_{n}=f_{2 r+1}+$ $3 \lambda+5$ if and only if the answer to the RPP instance is yes.

Before doing this, we note that the input size of this instance is polynomial of that of the RPP instance. To see this, let $L$ be the input size of the RPP instance. It follows immediately that $k=\mathcal{O}(L)$. We next show that the number of elements $n$ is satisfied with $n=\mathcal{O}(L)$. Since the input size of a positive integer $t$ is $\log _{2}(t+1)$, it follows that

$$
\log _{2}\left(2^{m+2}-2+1\right)=\log _{2}\left(\sum_{i \in K} \omega_{i}+1\right) \leq \sum_{i \in K} \log _{2}\left(\omega_{i}+1\right) \leq L,
$$

where the first inequality follows from $\omega_{i}+1 \geq 2$ for all $i \in K$. Thus, we have that $m \leq L-2$, which further implies that $r=m+6=\mathcal{O}(L)$. This, combined with the fact that $k=\mathcal{O}(L)$, indicates

$$
n=2 r+1+k=\mathcal{O}(L) .
$$

Finally, it can be easily verified that $a_{i}=\mathcal{O}\left(L^{2}\right)$ for $i=1, \ldots, n$ and $b=$ $\mathcal{O}\left(L^{2}\right)$. This proves that the input size of the constructed instance is polynomial of that of the RPP instance. For preparation of the proof, we also verify

$$
\begin{aligned}
f_{2 r+1} & \geq \frac{1}{4}(\sqrt{2}-1)(\sqrt{2})^{2 r+1}=\frac{1}{4}(2-\sqrt{2}) 2^{r}=\frac{1}{4}(2-\sqrt{2}) 2^{m+6} \\
& >2^{m+2}+4=2 \lambda+6 .
\end{aligned}
$$

In the following, we consider the lifting procedure. By construction, the knapsack inequality of this instance reads

$$
\begin{aligned}
& \sum_{i=1}^{2 r+1} \lambda f_{i} x_{i}+[\lambda(\lambda+3)+1] x_{2 r+2}+[\lambda(\lambda+3)-1] x_{2 r+3}+\sum_{i=2 r+4}^{n-1} \omega_{i}(\lambda+1) x_{i} \\
& \quad+\lambda\left(3 \lambda+6+f_{2 r+1}\right) x_{n} \leq \sum_{i=1}^{2 r+1} \lambda f_{i}+\lambda(3 \lambda+6) .
\end{aligned}
$$


Since $a_{1}=\lambda, a_{2}=\lambda$, and

$$
\begin{array}{r}
b-\sum_{i \in N_{1}} a_{i}=\sum_{i=1}^{2 r+1} \lambda f_{i}+\lambda(3 \lambda+6)-\sum_{i=1}^{r} \lambda f_{2 i+1}-\lambda\left(3 \lambda+6+f_{2 r+1}\right) \\
=\sum_{i=1}^{2 r} \lambda f_{i}-\sum_{i=1}^{r} \lambda f_{2 i+1}=\sum_{i=1}^{2 r} \lambda f_{i}-\lambda f_{3}-\sum_{i=2}^{r} \lambda\left(f_{2 i-1}+f_{2 i}\right)=\lambda,
\end{array}
$$

we know that $C$ is a minimal cover of $X\left(N_{0}, N_{1}\right)$ with the cover inequality

$$
x_{1}+x_{2} \leq 1 .
$$

\section{Step 1. Lifting the variables $x_{3}, \ldots, x_{2 r+1}$}

Starting with the cover inequality (11), we know from Observation 1 and the inequality (8) that, after lifting the variables $x_{3}, \ldots, x_{2 r+1}$, the inequality is

$$
\sum_{i=1}^{2 r+1} f_{i} x_{i} \leq \sum_{i=1}^{2 r} f_{i}
$$

\section{Step 2. Lifting the variables $x_{2 r+2}$ and $x_{2 r+3}$}

We first consider the variable $x_{2 r+2}$. The associated lifting problem is

$$
\begin{aligned}
\alpha_{2 r+2}=\min & \sum_{i=1}^{2 r} f_{i}-\sum_{i=1}^{2 r+1} f_{i} x_{i} \\
\text { s.t. } & \sum_{i=1}^{2 r+1} \lambda f_{i} x_{i} \leq \lambda \sum_{i=1}^{2 r} f_{i}-[\lambda(\lambda+3)+1], x \in \mathbb{B}^{2 r+1} .
\end{aligned}
$$

The problem (12) is feasible at the zero vector since

$$
\lambda \sum_{i=1}^{2 r} f_{i}-[\lambda(\lambda+3)+1] \geq \lambda\left(\sum_{i=1}^{2 r} f_{i}-\lambda-4\right)>\lambda\left(f_{2 r+1}-\lambda-4\right)>0,
$$

where the last inequality follows from (10). Let $\bar{x}$ be the optimal solution of (12). Since $f_{i} \in \mathbb{Z}$ for $i=1, \ldots, 2 r+1$, its feasibility requires

$$
\sum_{i=1}^{2 r+1} f_{i} \bar{x}_{i} \leq\left\lfloor\frac{\lambda \sum_{i=1}^{2 r} f_{i}-\lambda(\lambda+3)-1}{\lambda}\right\rfloor=\sum_{i=1}^{2 r} f_{i}-\lambda-4 .
$$

On the other hand, from Lemma 2, we can always find an $\bar{x}$ such that

$$
\sum_{i=1}^{2 r+1} f_{i} \bar{x}_{i}=\sum_{i=1}^{2 r} f_{i}-\lambda-4
$$


The optimality of $\bar{x}$ gives that $\alpha_{2 r+2}=\sum_{i=1}^{2 r} f_{i}-\sum_{i=1}^{2 r+1} f_{i} \bar{x}_{i}=\lambda+4$. Similarly, the lifting problem of $x_{2 r+3}$ reads

$$
\begin{aligned}
\alpha_{2 r+3}=\min & \sum_{i=1}^{2 r} f_{i}-\sum_{i=1}^{2 r+1} f_{i} x_{i}-(\lambda+4) x_{2 r+2} \\
\text { s.t. } & \sum_{i=1}^{2 r+1} \lambda f_{i} x_{i}+[\lambda(\lambda+3)+1] x_{2 r+2} \leq \lambda \sum_{i=1}^{2 r} f_{i}-[\lambda(\lambda+3)-1], \\
& x \in \mathbb{B}^{2 r+2} .
\end{aligned}
$$

Then $\alpha_{2 r+3}=\lambda+2$, which is achieved at an optimal solution $\bar{x}$ satisfying $\bar{x}_{2 r+2}=1$ and $\sum_{i=1}^{2 r+1} f_{i} \bar{x}_{i}=\sum_{i=1}^{2 r} f_{i}-2 \lambda-6$.

\section{Step 3. Lifting the variables $x_{2 r+4}, \ldots, x_{n-1}$}

We shall show that $\alpha_{i}=\omega_{i}$ for all $i \in K$ by induction. At first, consider the lifting of the variable $x_{2 r+4}$. This requires to solve the problem

$$
\begin{aligned}
\alpha_{2 r+4}=\min & \sum_{i=1}^{2 r} f_{i}-\sum_{i=1}^{2 r+1} f_{i} x_{i}-(\lambda+4) x_{2 r+2}-(\lambda+2) x_{2 r+3} \\
\text { s.t. } & \sum_{i=1}^{2 r+1} \lambda f_{i} x_{i}+[\lambda(\lambda+3)+1] x_{2 r+2}+[\lambda(\lambda+3)-1] x_{2 r+3} \\
& \leq \lambda \sum_{i=1}^{2 r} f_{i}-\omega_{2 r+4}(\lambda+1), x \in \mathbb{B}^{2 r+3} .
\end{aligned}
$$

If $\hat{x}$ is an optimal solution of the problem (14) with $\hat{x}_{2 r+3}=1$, by the feasibility and (10), we have

$$
\sum_{i=1}^{2 r+1} f_{i} \hat{x}_{i}+\lambda+2<\sum_{i=1}^{2 r} f_{i}+\lambda+2<\sum_{i=1}^{2 r+1} f_{i}
$$

This, together with Lemma 2, indicates that we can define a new feasible point $\bar{x}$ such that $\bar{x}_{2 r+2}=\hat{x}_{2 r+2}, \bar{x}_{2 r+3}=0$, and $\sum_{i=1}^{2 r+1} f_{i} \bar{x}_{i}=\sum_{i=1}^{2 r+1} f_{i} \hat{x}_{i}+\lambda+2$. It is easy to see that $\bar{x}$ and $\hat{x}$ give the same objective values. Hence, we can assume that $x_{2 r+3}=0$ in the problem (14). Furthermore, since $\omega_{2 r+4} \leq \lambda-1$, similar to the problem (13), we can show that the optimal value of (14) is $\alpha_{2 r+4}=\omega_{2 r+4}$, which is achieved at an optimal solution $\bar{x}$ satisfying $\bar{x}_{2 r+3}=0$, $\bar{x}_{2 r+2}=1$, and $\sum_{i=1}^{2 r+1} f_{i} \bar{x}_{i}=\sum_{i=1}^{2 r} f_{i}-(\lambda+4)-\omega_{2 r+4}$.

Now assume that $\alpha_{i}=\omega_{i}$ for $2 r+4 \leq i \leq j$ and $j<n-1$ and consider the lifting problem of $x_{j+1}$ : 


$$
\begin{aligned}
\alpha_{j+1}=\min & \sum_{i=1}^{2 r} f_{i}-\sum_{i=1}^{2 r+1} f_{i} x_{i}-(\lambda+4) x_{2 r+2}-(\lambda+2) x_{2 r+3}-\sum_{i=2 r+4}^{j} \omega_{i} x_{i} \\
\text { s.t. } \quad & \sum_{i=1}^{2 r+1} \lambda f_{i} x_{i}+[\lambda(\lambda+3)+1] x_{2 r+2}+[\lambda(\lambda+3)-1] x_{2 r+3}+ \\
& \sum_{i=2 r+4}^{j} \omega_{i}(\lambda+1) x_{i} \leq \lambda \sum_{i=1}^{2 r} f_{i}-\omega_{j+1}(\lambda+1), x \in \mathbb{B}^{j} .
\end{aligned}
$$

We claim that there exists an optimal solution $\bar{x}$ such that $\bar{x}_{2 r+3}=\bar{x}_{2 r+4}=$ $\cdots=\bar{x}_{j}=0$. To see this, suppose that an optimal solution $\hat{x}$ is such that $\hat{x}_{t}=1$ for some $t \subseteq[2 r+3, j]$. Analogously, define a new point $\bar{x}$ with $\bar{x}_{i}=\hat{x}_{i}$ for $2 r+2 \leq i \leq j$ and $i \neq t, \bar{x}_{t}=0$, and $\sum_{i=1}^{2 r+1} f_{i} \bar{x}_{i}=\sum_{i=1}^{2 r+1} f_{i} \hat{x}_{i}+\theta_{t}$, where

$$
\theta_{t}= \begin{cases}\lambda+2, & \text { if } t=2 r+3 \\ \omega_{t}, & \text { otherwise }\end{cases}
$$

By simple calculations, $\bar{x}$ is feasible to the problem (15) and gives the same objective value as $\bar{x}$. Similar to the problem (13), we can verify that $\alpha_{j+1}=$ $\omega_{j+1}$. Thus by induction, we have that $\alpha_{i}=\omega_{i}$ for all $i \in K$.

\section{Step 4. Lifting the variable $x_{n}$}

Finally, we concentrate on lifting the variable $x_{n}$. The lifting problem is

$$
\begin{aligned}
\beta_{n}=\max & \sum_{i=1}^{2 r+1} f_{i} x_{i}+(\lambda+4) x_{2 r+2}+(\lambda+2) x_{2 r+3}+\sum_{i=2 r+4}^{n-1} \omega_{i} x_{i}-\sum_{i=1}^{2 r} f_{i} \\
\text { s.t. } & \sum_{i=1}^{2 r+1} \lambda f_{i} x_{i}+[\lambda(\lambda+3)+1] x_{2 r+2}+[\lambda(\lambda+3)-1] x_{2 r+3}+ \\
& \sum_{i=2 r+4}^{n-1} \omega_{i}(\lambda+1) x_{i} \leq \lambda \sum_{i=1}^{2 r+1} f_{i}+\lambda(3 \lambda+6), x \in \mathbb{B}^{n-1} .
\end{aligned}
$$

For convenience, denote $g(x)$ to be the objective function in the above problem. Consider the point $\check{x}$ with $\check{x}_{i}=1$ for $2 r+2 \leq i \leq n-1$ and $\sum_{i=1}^{2 r+1} f_{i} \check{x}_{i}=$ $\sum_{i=1}^{2 r+1} f_{i}-\lambda-2$. By Lemma 2, such a point must exist. We can check that $\check{x}$ is feasible to the problem (16) and $g(\check{x})=f_{2 r+1}+3 \lambda+4$. Furthermore, for a binary vector $x$, if at least one of the two components $x_{2 r+2}$ and $x_{2 r+3}$ is equal to zero, we have that

$$
g(x) \leq \sum_{i=1}^{2 r+1} f_{i}+\lambda+4+\sum_{2 r+4}^{n-1} \omega_{i}-\sum_{i=1}^{2 r} f_{i}=f_{2 r+1}+3 \lambda+4
$$


Thus to seek better values for $\beta_{n}$, we may set $x_{2 r+2}=x_{2 r+3}=1$. In this case, the problem (16) reduces to

$$
\begin{aligned}
\beta_{n}= & \max \\
\text { s.t. } & \sum_{i=1}^{2 r+1} f_{i} x_{i}+\sum_{i=2 r+4}^{n-1} \omega_{i} x_{i}+2 \lambda+6-\sum_{i=1}^{2 r} f_{i} x_{i}+\sum_{i=2 r+4}^{n-1} \omega_{i}(\lambda+1) x_{i} \leq \lambda \sum_{i=1}^{2 r+1} f_{i}+\lambda^{2}, x \in \mathbb{B}^{n-3} .
\end{aligned}
$$

Now assume that $\bar{x}$ is an optimal solution of (17). Denote $p=\sum_{i=2 r+4}^{n-1} \omega_{i} \bar{x}_{i}$. It is easy to see that $p \leq 2 \lambda$. Consider the following four cases.

(a) $p \leq \lambda-2$. In this case, the knapsack constraint in the problem (17) is trivially satisfied and the optimality of $\bar{x}$ implies $\bar{x}_{i}=1$ for $i=1, \ldots, 2 r+1$. This further indicates that

$$
g(\bar{x})=\sum_{i=1}^{2 r+1} f_{i}+p+2 \lambda+6-\sum_{i=1}^{2 r} f_{i}=f_{2 r+1}+p+2 \lambda+6 \leq f_{2 r+1}+3 \lambda+4 .
$$

(b) $p=\lambda-1$. Similar to the case (a), we have that $g(\bar{x})=f_{2 r+1}+3 \lambda+5$.

(c) $p=\lambda$. In this case, the feasibility of $\bar{x}$ indicates that

$$
\sum_{i=1}^{2 r+1} f_{i} \bar{x}_{i} \leq\left[\lambda \sum_{i=1}^{2 r+1} f_{i}+\lambda^{2}-\lambda(\lambda+1)\right] / \lambda=\sum_{i=1}^{2 r+1} f_{i}-1
$$

Furthermore, the optimality of $\bar{x}$ implies that $\sum_{i=1}^{2 r+1} f_{i} \bar{x}_{i}=\sum_{i=1}^{2 r+1} f_{i}-1$. Thus we can also check that $g(\bar{x})=f_{2 r+1}+3 \lambda+5$.

(d) $\lambda+1 \leq p \leq 2 \lambda$. On one hand, the feasibility of $\bar{x}$ requires

$$
\sum_{i=1}^{2 r+1} f_{i} \bar{x}_{i} \leq\left\lfloor\frac{\lambda \sum_{i=1}^{2 r+1} f_{i}+\lambda^{2}-p(\lambda+1)}{\lambda}\right\rfloor=\sum_{i=1}^{2 r+1} f_{i}+\lambda-p-2,
$$

where the last equality follows from $\lambda+1 \leq p \leq 2 \lambda$. On the other hand, the optimality of $\bar{x}$ requires that the inequality in (18) holds with equality, yielding

$$
g(\bar{x})=\sum_{i=1}^{2 r+1} f_{i}+\lambda-p-2+p+2 \lambda+6-\sum_{i=1}^{2 r} f_{i}=f_{2 r+1}+3 \lambda+4 .
$$

To summarize, the lifting coefficient $\beta_{n}=f_{2 r+1}+3 \lambda+5$ for the problem of computing the sequential LCI constructed in the above, if any only if $p=\lambda$ or $p=\lambda-1$, or equivalently, the answer to the RPP instance is yes. Since the RPP problem is $\mathcal{N} \mathcal{P}$-hard and the construction of the companion problem is in polynomial, we conclude that computing a sequential LCI is $\mathcal{N} \mathcal{P}$-hard. This completes the proof. 


\section{References}

1. Atamtürk, A.: Cover and pack inequalities for (mixed) integer programming. Annals of Operations Research 139(1), 21-38 (2005)

2. Atamtürk, A., Rajan, D.: On splittable and unsplittable flow capacitated network design arc-set polyhedra. Mathematical Programming 92(2), 315333 (2002)

3. Balas, E.: Facets of the knapsack polytope. Mathematical Programming 8(1), 146-164 (1975)

4. Balas, E., Zemel, E.: Facets of the Knapsack Polytope From Minimal Covers. SIAM Journal on Applied Mathematics 34(1), 119-148 (1978)

5. Conforti, M., Cornuéjols, G., Zambelli, G.: Integer programming. Springer (2014)

6. Crowder, H., Johnson, E.L., Padberg, M.: Solving large-scale zero-one linear programming problems. Operations Research 31(5), 803-834 (1983)

7. Garey, M.R., Johnson, D.: Computers and Intractability: A Guide to the Theory of NP-Completeness. W.H. Freeman and Company, New York (1979)

8. Gu, Z.: Lifted cover inequalities for $0-1$ and mixed $0-1$ integer programs. Ph.D. thesis, Georgia Institute of Technology (1995)

9. Gu, Z., Nemhauser, G.L., Savelsbergh, M.W.: Lifted cover inequalities for 0-1 integer programs: Complexity. INFORMS Journal on Computing 11(1), 117-123 (1999)

10. Gu, Z., Nemhauser, G.L., Savelsbergh, M.W.P.: Lifted cover inequalities for 0-1 integer programs: Computation. INFORMS Journal on Computing $\mathbf{1 0}(4), 427-437$ (1998)

11. Gu, Z., Nemhauser, G.L., Savelsbergh, M.W.P.: Sequence Independent Lifting in Mixed Integer Programming. Journal of Combinatorial Optimization 4(1), 109-129 (2000)

12. Gupta, S.R., Bulfin, R.L., Smith, J.S.: Lifting cover inequalities for the binary knapsack polytope (2005). http://citeseerx.ist.psu.edu/viewdoc/summary?doi=10.1.1.83.9230

13. Hammer, P.L., Johnson, E.L., Peled, U.N.: Facet of regular 0-1 polytopes. Mathematical Programming 8(1), 179-206 (1975)

14. Hartvigsen, D., Zemel, E.: The complexity of lifted inequalities for the knapsack problem. Discrete Applied Mathematics 39(2), 113-123 (1992)

15. Hoffman, K.L., Padberg, M.: Improving LP-representations of zero-one linear programs for branch-and-cut. ORSA Journal on Computing 3(2), 121-134 (1991)

16. Kaparis, K., Letchford, A.N.: Separation algorithms for 0-1 knapsack polytopes. Mathematical Programming 124(1-2), 69-91 (2010)

17. Kaparis, K., Letchford, A.N.: Cover inequalities. In: Wiley Encyclopedia of Operations Research and Management Science, pp. 1074-1080 (2011)

18. Klabjan, D., Nemhauser, G.L., Tovey, C.: The complexity of cover inequality separation. Operations Research Letters 23(1-2), 35-40 (1998) 
19. Letchford, A.N., Souli, G.: On lifted cover inequalities: A new lifting procedure with unusual properties. Operations Research Letters 47(2), 83-87 (2019)

20. Nemhauser, G.L., Wolsey, L.A.: Integer and combinatorial optimization. Wiley-Interscience (1988)

21. Padberg, M.W.: Technical NoteA Note on Zero-One Programming. Operations Research 23(4), 833-837 (1975)

22. Van Hoesel, S.P.M., Koster, A.M.C.A., Van De Leensel, R.L.M.J., Savelsbergh, M.W.P.: Polyhedral results for the edge capacity polytope. Mathematical Programming 92(2), 335-358 (2002)

23. Van Roy, T.J., Wolsey, L.A.: Solving mixed integer programming problems using automatic reformulation. Operations Research 35(1), 45-57 (1987)

24. Wolsey, L.A.: Faces for a linear inequality in 0-1 variables. Mathematical Programming 8(1), 165-178 (1975)

25. Wolter, K.: Implementation of cutting plane separators for mixed integer programs. Diploma thesis, Technische Universität Berlin (2006)

26. Zemel, E.: Easily computable facets of the knapsack polytope. Mathematics of Operations Research 14(4), 760-764 (1989) 